\title{
Inclusion Properties for Certain Subclasses of Analytic Functions Defined by a Linear Operator
}

\author{
Nak Eun Cho \\ Department of Applied Mathematics, Pukyong National University, Pusan 608-737, South Korea \\ Correspondence should be addressed to Nak Eun Cho, necho@pknu.ac.kr
}

Received 8 August 2007; Revised 29 October 2007; Accepted 23 November 2007

Recommended by John Michael Rassias

The purpose of the present paper is to investigate some inclusion properties of certain subclasses of analytic functions associated with a family of linear operators, which are defined by means of the Hadamard product (or convolution). Some integral preserving properties are also considered.

Copyright (C) 2008 Nak Eun Cho. This is an open access article distributed under the Creative Commons Attribution License, which permits unrestricted use, distribution, and reproduction in any medium, provided the original work is properly cited.

\section{Introduction}

Let $\mathcal{A}$ denote the class of functions of the form

$$
f(z)=z+\sum_{k=2}^{\infty} a_{k} z^{k}
$$

which are analytic in the open unit disk $\mathbb{U}=\{z \in \mathbb{C}:|z|<1\}$. If $f$ and $g$ are analytic in $\mathbb{U}$, we say that $f$ is subordinate to $g$, written $f<g$ or $f(z) \prec g(z)$ if there exists an analytic function $w$ in $\mathbb{U}$ with $w(0)=0$ and $|w(z)|<1$ for $z \in \mathbb{U}$ such that $f(z)=g(w(z))$. We denote by $\mathcal{S}^{*}, \mathcal{K}$, and $\mathcal{C}$ the subclasses of $\mathcal{A}$ consisting of all analytic functions which are, respectively, starlike, convex, and close-to-convex in $\mathbb{U}$.

Let $\mathcal{N}$ be the class of all functions $\phi$ which are analytic and univalent in $\mathbb{U}$ and for which $\phi(\mathbb{U})$ is convex with $\phi(0)=1$ and $\operatorname{Re}\{\phi(z)\}>0$ for $z \in \mathbb{U}$.

Making use of the principle of subordination between analytic functions, many authors investigated the subclasses $\mathcal{S}^{*}(\phi), \mathcal{K}(\phi)$, and $\mathcal{C}(\phi, \psi)$ of the class $\mathcal{A}$ for $\phi, \psi \in \mathcal{N}($ cf. $[1,2])$, which are defined by

$$
\mathcal{S}^{*}(\phi):=\left\{f \in \mathcal{A}: \frac{z f^{\prime}(z)}{f(z)} \prec \phi(z) \text { in } \mathbb{U}\right\}
$$




$$
\begin{aligned}
\mathcal{K}(\phi) & :=\left\{f \in \mathcal{A}: 1+\frac{z f^{\prime \prime}(z)}{f^{\prime}(z)} \prec \phi(z) \text { in } \mathbb{U}\right\}, \\
\mathcal{C}(\phi, \psi) & :=\left\{f \in \mathcal{A}: \exists g \in \mathcal{S}^{*}(\phi) \text { s.t. } \frac{z f^{\prime}(z)}{g(z)} \prec \psi(z) \text { in } \mathbb{U}\right\} .
\end{aligned}
$$

For $\phi(z)=\psi(z)=(1+z) /(1-z)$ in the definitions defined above, we have the well-known classes $\mathcal{S}^{*}, \mathcal{K}$, and $\mathcal{C}$, respectively. Furthermore, for the function classes $\mathcal{S}^{*}[A, B]$ and $\mathcal{K}[A, B]$ investigated by Janowski [3] (also see [4]), it is easily seen that

$$
\begin{aligned}
& \mathcal{S}^{*}\left(\frac{1+A z}{1+B z}\right)=\mathcal{S}^{*}[A, B] \quad(-1 \leq B<A \leq 1), \\
& \mathcal{K}\left(\frac{1+A z}{1+B z}\right)=\mathcal{K}[A, B] \quad(-1 \leq B<A \leq 1) .
\end{aligned}
$$

We now define the function $h(a, c)(z)$ by

$$
h(a, c)(z):=\sum_{k=0}^{\infty} \frac{(a)_{k}}{(c)_{k}} z^{k+1}, \quad\left(z \in \mathbb{U} ; a \in \mathbb{R} ; c \in \mathbb{R} \backslash \mathbb{Z}_{0}^{-} ; \mathbb{Z}_{0}^{-}:=\{0,-1,-2, \ldots\}\right),
$$

where $(v)_{k}$ is the Pochhammer symbol (or the shifted factorial) defined (in terms of the Gamma function) by

$$
(v)_{k}:=\frac{\Gamma(v+k)}{\Gamma(v)}= \begin{cases}1 & \text { if } k=0, v \in \mathbb{C} \backslash\{0\}, \\ v(v+1) \cdots(v+k-1) & \text { if } k \in \mathbb{N}:=\{1,2, \ldots\}, v \in \mathbb{C} .\end{cases}
$$

We also denote by $L(a, c): \mathcal{A} \rightarrow \mathcal{A}$ the operator defined by

$$
L(a, c) f(z)=h(a, c)(z) * f(z) \quad(z \in \mathbb{U} ; f \in \mathcal{A}),
$$

where the symbol $(*)$ stands for the Hadamard product (or convolution). Then it is easily observed from definitions (1.4) and (1.6) that $L(2,1) f(z)=z f^{\prime}(z)$ and

$$
z(L(a, c) f(z))^{\prime}=a L(a+1, c) f(z)-(a-1) L(a, c) f(z) .
$$

Furthermore, we note that $L(n+1,1) f(z)=D^{n} f(z)(n>-1)$, where the symbol $D^{n}$ denotes the familiar Ruscheweyh derivative [5] (also, see [6]) for $n \in \mathbb{N}_{0}:=\mathbb{N} \cup\{0\}$. The operator $L(a, c)$ was introduced and studied by Carlson and Shaffer [7] which has been used widely on the space of analytic and univalent functions in $\mathbb{U}$ (see also [8]).

By using the operator $L(a, c)$, we introduce the following classes of analytic functions for $\phi, \psi \in \mathcal{N}, a \in \mathbb{R}$ and $c \in \mathbb{R} \backslash \mathbb{Z}_{0}^{-}$:

$$
\begin{aligned}
\mathcal{S}_{a, c}(\phi) & :=\left\{f \in \mathcal{A}: L(a, c) f(z) \in \mathcal{S}^{*}(\phi)\right\}, \\
\mathcal{K}_{a, c}(\phi) & :=\{f \in \mathcal{A}: L(a, c) f(z) \in \mathcal{K}(\phi)\}, \\
\mathcal{C}_{a, c}(\phi, \psi) & :=\{f \in \mathcal{A}: L(a, c) f(z) \in \mathcal{C}(\phi, \psi)\} .
\end{aligned}
$$


We also note that

$$
f(z) \in \mathcal{K}_{a, c}(\phi) \Longleftrightarrow z f^{\prime}(z) \in \mathcal{S}_{a, c}(\phi)
$$

In particular, we set

$$
\begin{aligned}
& \mathcal{S}_{a, c}\left(\frac{1+A z}{1+B z}\right)=\mathcal{S}_{a, c}[A, B] \quad(-1 \leq B<A \leq 1), \\
& \mathcal{K}_{a, c}\left(\frac{1+A z}{1+B z}\right)=\mathcal{K}_{a, c}[A, B] \quad(-1 \leq B<A \leq 1) .
\end{aligned}
$$

In this paper, we investigate several inclusion properties of the classes $\mathcal{S}_{a, c}(\phi), \mathcal{K}_{a, c}(\phi)$, and $\mathcal{C}_{a, c}(\phi, \psi)$. The integral preserving properties in connection with the operator $L(a, c)$ are also considered. Furthermore, relevant connections of the results presented here with those obtained in earlier works are pointed out.

\section{Inclusion properties involving the operator $L(a, c)$}

The following lemmas will be required in our investigation.

Lemma 2.1 (see [9, pages 60-61]). Let $a_{2} \geq a_{1}>0$. If $a_{2} \geq 2$ or $a_{1}+a_{2} \geq 3$, then the function $h\left(a_{1}, a_{2}\right)(z)$ defined by (1.4) belongs to the class $\mathcal{K}$.

Lemma 2.2 (see [10]). Let $f \in \mathcal{K}$ and $g \in \mathcal{S}^{*}$. Then for every analytic function $Q$ in $\mathbb{U}$,

$$
\frac{(f * Q g)}{(f * g)}(\mathbb{U}) \subset \overline{\operatorname{co}} Q(\mathbb{U})
$$

where $\overline{\operatorname{co}} Q(\mathbb{U})$ denote the closed convex hull of $Q(\mathbb{U})$.

Theorem 2.3. Let $a_{2} \geq a_{1}>0, c \in \mathbb{R} \backslash \mathbb{Z}_{0}^{-}$, and $\phi \in \mathcal{N}$. If $a_{2} \geq 2$ or $a_{1}+a_{2} \geq 3$, then

$$
\mathcal{S}_{a_{2}, c}(\phi) \subset \mathcal{S}_{a_{1}, c}(\phi) \text {. }
$$

Proof. Let $f \in \mathcal{S}_{a_{2}, c}(\phi)$. Then there exists an analytic function $w$ in $\mathbb{U}$ with $|w(z)|<1(z \in \mathbb{U})$ and $w(0)=0$ such that

$$
\frac{z\left(L\left(a_{2}, c\right) f(z)\right)^{\prime}}{L\left(a_{2}, c\right) f(z)}=\phi(w(z)) \quad(z \in \mathbb{U}) .
$$

By using (1.6) and (2.3), we have

$$
\begin{aligned}
\frac{z\left(L\left(a_{1}, c\right) f(z)\right)^{\prime}}{L\left(a_{1}, c\right) f(z)} & =\frac{z\left(h\left(a_{1}, c\right)(z) * f(z)\right)^{\prime}}{h\left(a_{1}, c\right)(z) * f(z)} \\
& =\frac{z\left(h\left(a_{2}, c\right)(z) * h\left(a_{1}, a_{2}\right)(z) * f(z)\right)^{\prime}}{h\left(a_{2}, c\right)(z) * h\left(a_{1}, a_{2}\right)(z) * f(z)} \\
& =\frac{h\left(a_{1}, a_{2}\right)(z) * z\left(L\left(a_{2}, c\right) f(z)\right)^{\prime}}{h\left(a_{1}, a_{2}\right)(z) * L\left(a_{2}, c\right) f(z)} \\
& =\frac{h\left(a_{1}, a_{2}\right)(z) * \phi(w(z)) L\left(a_{2}, c\right) f(z)}{h\left(a_{1}, a_{2}\right)(z) * L\left(a_{2}, c\right) f(z)}
\end{aligned}
$$


It follows from (2.3) and Lemma 2.1 that $L\left(a_{2}, c\right) f(z) \in \mathcal{S}^{*}$ and $h\left(a_{1}, a_{2}\right)(z) \in \mathcal{K}$, respectively. Then by applying Lemma 2.2 to (2.4), we obtain

$$
\frac{\left\{h\left(a_{1}, a_{2}\right)(z) * \phi(w) L\left(a_{2}, c\right) f\right\}}{\left\{h\left(a_{1}, a_{2}\right)(z) * L\left(a_{2}, c\right) f\right\}}(\mathbb{U}) \subset \overline{\mathrm{co}}(\phi(\mathbb{U})) \subset \phi(\mathbb{U}),
$$

since $\phi$ is convex univalent. Therefore, from the definition of subordination and (2.5), we have

$$
\frac{z\left(L\left(a_{1}, c\right) f(z)\right)^{\prime}}{L\left(a_{1}, c\right) f(z)} \prec \phi(z) \quad(z \in \mathbb{U})
$$

or, equivalently, $f \in \mathcal{S}_{a_{1}, c}(\phi)$, which completes the proof of Theorem 2.3.

Theorem 2.4. Let $a \in \mathbb{R}, c_{2} \geq c_{1}>0$ and $\phi \in \mathcal{N}$. If $c_{2} \geq 2$ or $c_{1}+c_{2} \geq 3$, then

$$
\mathcal{S}_{a, c_{1}}(\phi) \subset \mathcal{S}_{a, c_{2}}(\phi)
$$

Proof $\left(f \in \mathcal{S}_{a, c_{1}}(\phi)\right)$. Using a similar argument as in the proof of Theorem 2.3, we obtain

$$
\frac{z\left(L\left(a, c_{2}\right) f(z)\right)^{\prime}}{L\left(a, c_{2}\right) f(z)}=\frac{h\left(a_{1}, a_{2}\right)(z) * \phi(w(z)) L\left(a, c_{1}\right) f(z)}{h\left(a_{1}, a_{2}\right)(z) * L\left(a, c_{1}\right) f(z)}
$$

where $w$ is an analytic function in $\mathbb{U}$ with $|w(z)|<1(z \in \mathbb{U})$ and $w(0)=0$. Applying Lemma 2.1 and the fact that $L\left(a, c_{1}\right) f(z) \in \mathcal{S}^{*}$, we see that

$$
\frac{\left\{h\left(a_{1}, a_{2}\right) * h(w) L\left(a_{2}, c\right) f\right\}}{\left\{h\left(a_{1}, a_{2}\right) * L\left(a, c_{1}\right) f\right\}}(\mathbb{U}) \subset \overline{\mathrm{co}}(\phi(\mathbb{U})) \subset \phi(\mathbb{U}),
$$

since $\phi$ is convex univalent. Thus the proof of Theorem 2.3 is completed.

Corollary 2.5. Let $a_{2} \geq a_{1}>0, c_{2} \geq c_{1}>0$, and $\phi \in \mathcal{N}$. If $a_{2} \geq \min \left\{2,3-a_{1}\right\}$ and $c_{2} \geq \min \left\{2,3-c_{1}\right\}$, then

$$
\mathcal{S}_{a_{2}, c_{1}}(\phi) \subset \mathcal{S}_{a_{2}, c_{2}}(\phi) \subset \mathcal{S}_{a_{1}, c_{2}}(\phi)
$$

Theorem 2.6. Let $a_{2} \geq a_{1}>0, c_{2} \geq c_{1}>0$ and $\phi \in \mathcal{N}$. If $a_{2} \geq \min \left\{2,3-a_{1}\right\}$ and $c_{2} \geq \min \left\{2,3-c_{1}\right\}$, then

$$
\mathcal{K}_{a_{2}, c_{1}}(\phi) \subset \mathcal{K}_{a_{2}, c_{2}}(\phi) \subset \mathcal{K}_{a_{1}, c_{2}}(\phi) .
$$


Proof. Applying (1.9) and Corollary 2.5, we observe that

$$
\begin{aligned}
f(z) \in \mathcal{K}_{a_{2}, c_{1}}(\phi) & \Longleftrightarrow L\left(a_{2}, c_{1}\right) f(z) \in \mathcal{K}(\phi) \\
& \Longleftrightarrow z\left(L\left(a_{2}, c_{1}\right) f(z)\right)^{\prime} \in \mathcal{S}^{*}(\phi) \\
& \Longleftrightarrow L\left(a_{2}, c_{1}\right)\left(z f^{\prime}(z)\right) \in \mathcal{S}^{*}(\phi) \\
& \Longleftrightarrow z f^{\prime}(z) \in \mathcal{S}_{a_{2}, c_{1}}(\phi) \\
& \Longleftrightarrow z f^{\prime}(z) \in \mathcal{S}_{a_{2}, c_{2}}(\phi) \\
& \Longleftrightarrow L\left(a_{2}, c_{2}\right)\left(z f^{\prime}(z)\right) \in \mathcal{S}^{*}(\phi) \\
& \Longleftrightarrow z\left(L\left(a_{2}, c_{2}\right) f(z)\right)^{\prime} \in \mathcal{S}^{*}(\phi) \\
& \Longleftrightarrow L\left(a_{2}, c_{2}\right) f(z) \in \mathcal{K}(\phi) \\
& \Longleftrightarrow f(z) \in \mathcal{K}_{a_{2}, c_{2}}(\phi), \\
f(z) \in \mathcal{K}_{a_{2}, c_{2}}(\phi) & \Longleftrightarrow L\left(a_{2}, c_{2}\right) f(z) \in \mathcal{K}(\phi) \\
& \Longleftrightarrow L\left(a_{2}, c_{2}\right)\left(z f^{\prime}(z)\right) \in \mathcal{S}^{*}(\phi) \\
& \Longleftrightarrow z f^{\prime}(z) \in \mathcal{S}_{a_{1}, c_{2}}(\phi) \\
& \Longleftrightarrow z\left(L\left(a_{1}, c_{2}\right) f(z)\right)^{\prime} \in \mathcal{S}^{*}(\phi) \\
& \Longleftrightarrow f(z) \in \mathcal{K}_{a_{1}, c_{2}}(\phi),
\end{aligned}
$$

which evidently proves Theorem 2.6.

Taking $\phi(z)=(1+A z) /(1+B z)(-1 \leq B<A \leq 1 ; z \in \mathbb{U})$ in Corollary 2.5 and Theorem 2.6, we have the following corollary.

Corollary 2.7. Let $a_{2} \geq a_{1}>0$ and $c_{2} \geq c_{1}>0$. If $a_{2} \geq \min \left\{2,3-a_{1}\right\}$ and $c_{2} \geq \min \left\{2,3-c_{1}\right\}$, then

$$
\begin{aligned}
& \mathcal{S}_{a_{2}, c_{1}}[A, B] \subset \mathcal{S}_{a_{2}, c_{2}}[A, B] \subset \mathcal{S}_{a_{1}, c_{2}}[A, B] \quad(-1 \leq B<A \leq 1), \\
& \mathcal{K}_{a_{2}, c_{1}}[A, B] \subset \mathcal{K}_{a_{2}, c_{2}}[A, B] \subset \mathcal{K}_{a_{1}, c_{2}}[A, B] \quad(-1 \leq B<A \leq 1) .
\end{aligned}
$$

To prove the theorems below, we need the following lemma.

Lemma 2.8. Let $\phi \in \mathcal{N}$. If $f \in \mathcal{K}$ and $q \in \mathcal{S}^{*}(\phi)$, then $f * q \in \mathcal{S}^{*}(\phi)$.

Proof. Let $q \in \mathcal{S}^{*}(\phi)$. Then

$$
z q^{\prime}(z)=q(z) \phi(\omega(z)) \quad(z \in \mathbb{U})
$$

where $\omega$ is an analytic function in $\mathbb{U}$ with $|w(z)|<1(z \in \mathbb{U})$ and $w(0)=0$. Thus we have

$$
\frac{z(f(z) * q(z))^{\prime}}{f(z) * q(z)}=\frac{f(z) * z q^{\prime}(z)}{f(z) * q(z)}=\frac{f(z) * \phi(\omega(z)) q(z)}{f(z) * q(z)} \quad(z \in \mathbb{U}) .
$$

By using similar arguments to those used in the proof of Theorem 2.3, we conclude that (2.15) is subordinated to $\phi$ in $\mathbb{U}$ and so $f * q \in \mathcal{S}^{*}(\phi)$. 
Theorem 2.9. Let $a_{2} \geq a_{1}>0, c_{2} \geq c_{1}>0$ and $\phi, \psi \in \mathcal{N}$. If $a_{2} \geq \min \left\{2,3-a_{1}\right\}$ and $c_{2} \geq$ $\min \left\{2,3-c_{1}\right\}$, then

$$
\mathcal{C}_{a_{2}, c_{1}}(\phi, \psi) \subset \mathcal{C}_{a_{2}, c_{2}}(\phi, \psi) \subset \mathcal{C}_{a_{1}, c_{2}}(\phi, \psi) .
$$

Proof. First of all, we show that

$$
\mathcal{C}_{a_{2}, c_{1}}(\phi, \psi) \subset \mathcal{C}_{a_{2}, c_{2}}(\phi, \psi) .
$$

Let $f \in \mathcal{C}_{a_{2}, c_{1}}(\phi, \psi)$. Then there exists a function $q_{2} \in \mathcal{S}^{*}(\phi)$ such that

$$
\frac{z\left(L\left(a_{2}, c_{1}\right) f(z)\right)^{\prime}}{q_{2}(z)} \prec \psi(z) \quad(z \in \mathbb{U}) .
$$

From (2.18), we obtain

$$
z\left(L\left(a_{2}, c_{1}\right) f(z)\right)^{\prime}=\psi(w(z)) \quad(z \in \mathbb{U}),
$$

where $w$ is an analytic function in $\mathbb{U}$ with $|w(z)|<1(z \in \mathbb{U})$ and $w(0)=0$. By virtue of Lemmas 2.1 and 2.8 , we see that $h\left(a_{1}, a_{2}\right)(z) * q_{2}(z) \equiv q_{1}(z)$ belongs to $\mathcal{S}^{*}(\phi)$. Then we have

$$
\begin{aligned}
\frac{z\left(L\left(a_{2}, c_{2}\right) f(z)\right)^{\prime}}{q_{1}(z)} & =\frac{h\left(c_{1}, c_{2}\right)(z) * z\left(L\left(a_{2}, c_{1}\right) f(z)\right)^{\prime}}{h\left(c_{1}, c_{2}\right)(z) * q_{2}(z)} \\
& =\frac{h\left(c_{1}, c_{2}\right)(z) * \psi(w(z)) q_{2}(z)}{h\left(c_{1}, c_{2}\right)(z) * q_{2}(z)} \\
& \prec \psi(z) \quad(z \in \mathbb{U}),
\end{aligned}
$$

which implies that $f \in \mathcal{C}_{a_{1}, c}(\phi, \psi)$.

Moreover, the proof of the second part is similar to that of the first part and so we omit the details involved.

\section{Inclusion properties involving various operators}

The next theorem shows that the classes $\mathcal{S}_{a, c}(\phi), \mathcal{K}_{a, c}(\phi)$, and $\mathcal{C}_{a, c}(\phi, \psi)$ are invariant under convolution with convex functions.

Theorem 3.1. Let $a>0, c \in \mathbb{R} \backslash \mathbb{Z}_{0}^{-}, \phi, \psi \in \mathcal{N}$ and let $g \in \mathcal{K}$. Then

(i) $f \in \mathcal{S}_{a, c}(\phi) \Rightarrow g * f \in \mathcal{S}_{a, c}(\phi)$,

(ii) $f \in \mathcal{K}_{a, c}(\phi) \Rightarrow g * f \in \mathcal{K}_{a, c}(\phi)$,

(iii) $f \in \mathcal{C}_{a, c}(\phi, \psi) \Rightarrow g * f \in \mathcal{C}_{a, c}(\phi, \psi)$.

Proof. (i) Let $f \in \mathcal{S}_{a, c}(\phi)$. Then we have

$$
\frac{z(L(a, c)(g * f)(z))^{\prime}}{L(a, c)(g * f)(z)}=\frac{g(z) * z(L(a, c) f(z))^{\prime}}{g(z) * L(a, c) f(z)} .
$$

By using the same techniques as in the proof of Theorem 2.3, we obtain (i). 
(ii) Let $f \in \mathcal{K}_{a, c}(\phi)$. Then, by (1.9), $z f^{\prime}(z) \in \mathcal{S}_{a, c}(\phi)$ and hence from (i), $g(z) * z f^{\prime}(z) \in$ $\mathcal{S}_{a, c}(\phi)$. Since

$$
g(z) * z f^{\prime}(z)=z(g * f)^{\prime}(z)
$$

we have (ii) applying (1.9) once again.

(iii) Let $f \in \mathcal{C}_{a, c}(\phi, \psi)$. Then there exists a function $q \in \mathcal{S}^{*}(\phi)$ such that

$$
z(L(a, c) f(z))^{\prime}=\psi(w(z)) q(z) \quad(z \in \mathbb{U})
$$

where $w$ is an analytic function in $\mathbb{U}$ with $|w(z)|<1(z \in \mathbb{U})$ and $w(0)=0$. From Lemma 2.8 , we have that $g * q \in \mathcal{S}^{*}(\phi)$. Since

$$
\frac{z(L(a, c)(g * f)(z))^{\prime}}{(g * q)(z)}=\frac{g(z) * z(L(a, c) f(z))^{\prime}}{g(z) * q(z)}=\frac{g(z) * \psi(w(z)) q(z)}{g(z) * q(z)} \prec \psi(z) \quad(z \in \mathbb{U}),
$$

we obtain (iii).

Now we consider the following operators $[5,11]$ defined by

$$
\begin{aligned}
& \Psi_{1}(z)=\sum_{k=1}^{\infty} \frac{1+c}{k+c} z^{k} \quad(\operatorname{Re}\{c\} \geq 0 ; z \in \mathbb{U}) \\
& \Psi_{2}(z)=\frac{1}{1-x} \log \left[\frac{1-x z}{1-z}\right] \quad(\log 1=0 ;|x| \leq 1, x \neq 1 ; z \in \mathbb{U}) .
\end{aligned}
$$

It is well known ([12], see also [5]) that the operators $\Psi_{1}$ and $\Psi_{2}$ are convex univalent in $\mathbb{U}$. Therefore, we have the following result, which can be obtained from Theorem 3.1 immediately.

Corollary 3.2. Let $a>0, c \in \mathbb{R} \backslash \mathbb{Z}_{0}^{-}, \phi, \psi \in \mathcal{N}$ and let $\Psi_{i}(i=1,2)$ be defined by (3.5). Then

(i) $f \in \mathcal{S}_{a, c}(\phi) \Rightarrow \Psi_{i} * f \in \mathcal{S}_{a, c}(\phi)$,

(ii) $f \in \mathcal{K}_{a, c}(\phi) \Rightarrow \Psi_{i} * f \in \mathcal{K}_{a, c}(\phi)$,

(iii) $f \in \mathcal{C}_{a, c}(\phi, \psi) \Rightarrow \Psi_{i} * f \in \mathcal{C}_{a, c}(\phi, \psi)$.

\section{Acknowledgments}

The author would like to express his gratitude to the referees for their valuable suggestions. This work was supported by Pukyong National University Research Fund in 2007 (PK-2007013).

\section{References}

[1] J. H. Choi, M. Saigo, and H. M. Srivastava, "Some inclusion properties of a certain family of integral operators," Journal of Mathematical Analysis and Applications, vol. 276, no. 1, pp. 432-445, 2002.

[2] W. Ma and D. Minda, "An internal geometric characterization of strongly starlike functions," Annales Universitatis Mariae Curie-Skłodowska. Sectio A, vol. 45, pp. 89-97, 1991.

[3] W. Janowski, "Some extremal problems for certain families of analytic functions. I," Bulletin de l'Académie Polonaise des Sciences. Série des Sciences Mathématiques, Astronomiques et Physiques, vol. 21, pp. 17-25, 1973. 
[4] R. M. Goel and B. S. Mehrok, "On the coefficients of a subclass of starlike functions," Indian Journal of Pure and Applied Mathematics, vol. 12, no. 5, pp. 634-647, 1981.

[5] St. Ruscheweyh, "New criteria for univalent functions," Proceedings of the American Mathematical Society, vol. 49, pp. 109-115, 1975.

[6] H. S. Al-Amiri, "On Ruscheweyh derivatives," Annales Polonici Mathematici, vol. 38, no. 1, pp. 88-94, 1980.

[7] B. C. Carlson and D. B. Shaffer, "Starlike and prestarlike hypergeometric functions," SIAM Journal on Mathematical Analysis, vol. 15, no. 4, pp. 737-745, 1984.

[8] H. M. Srivastava and S. Owa, "Some characterization and distortion theorems involving fractional calculus, generalized hypergeometric functions, Hadamard products, linear operators, and certain subclasses of analytic functions," Nagoya Mathematical Journal, vol. 106, pp. 1-28, 1987.

[9] St. Ruscheweyh, Convolutions in Geometric Function Theory, vol. 83 of Séminaire de Mathématiques Supérieures, Presses de l'Université de Montréal, Montreal, Quebec, Canada, 1982.

[10] St. Ruscheweyh and T. Sheil-Small, "Hadamard products of Schlicht functions and the PólyaSchoenberg conjecture," Commentarii Mathematici Helvetici, vol. 48, no. 1, pp. 119-135, 1973.

[11] S. Owa and H. M. Srivastava, "Some applications of the generalized Libera integral operator," Proceedings of the Japan Academy. Series A, vol. 62, no. 4, pp. 125-128, 1986.

[12] R. W. Barnard and Ch. Kellogg, "Applications of convolution operators to problems in univalent function theory," Michigan Mathematical Journal, vol. 27, no. 1, pp. 81-94, 1980. 


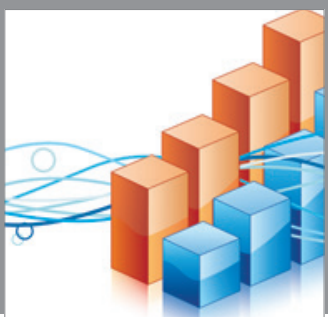

Advances in

Operations Research

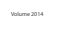

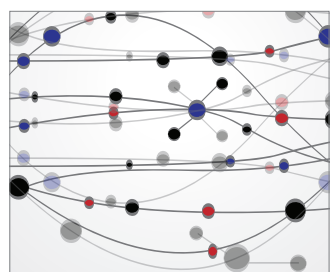

\section{The Scientific} World Journal
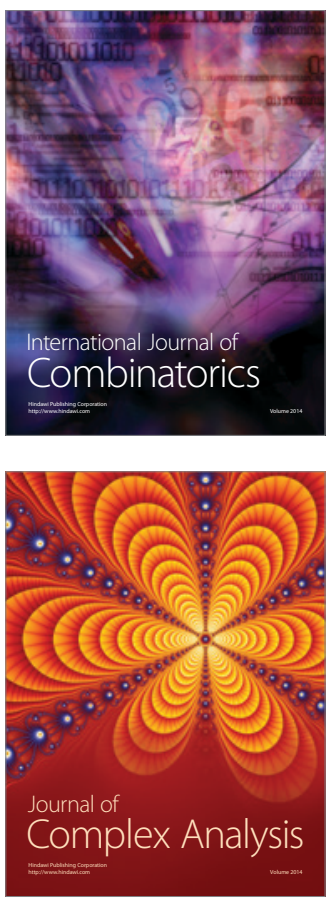

International Journal of

Mathematics and

Mathematical

Sciences
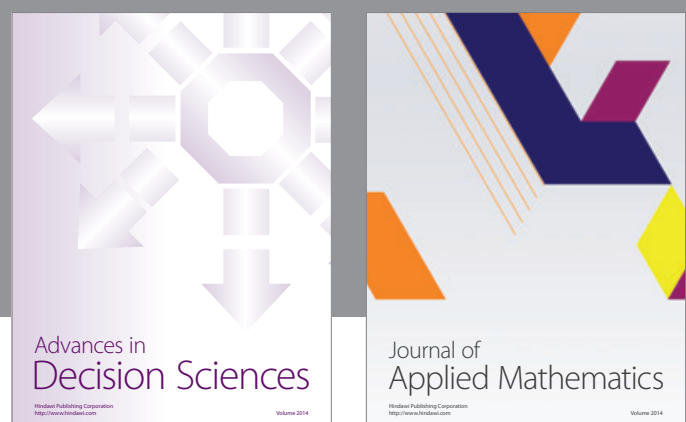

Journal of

Applied Mathematics
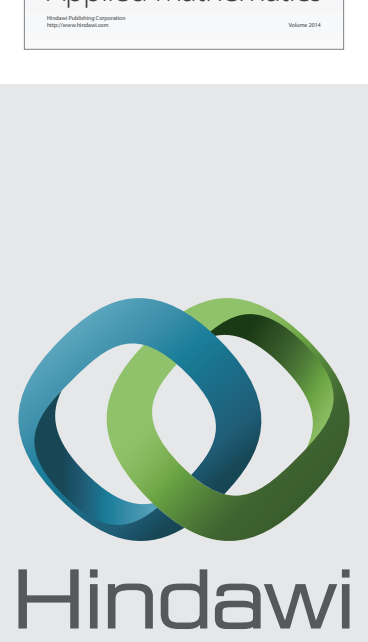

Submit your manuscripts at http://www.hindawi.com
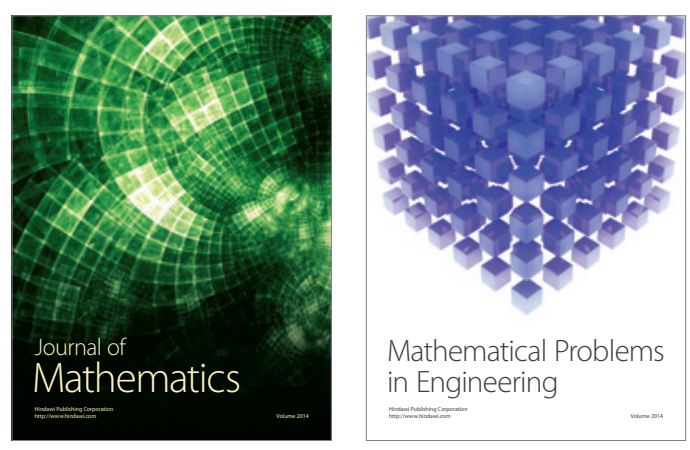

Mathematical Problems in Engineering
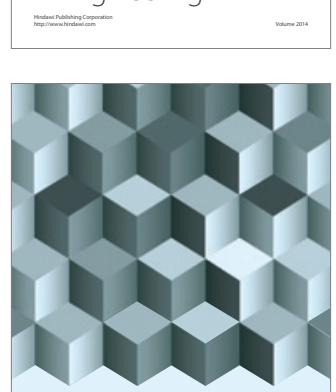

Journal of

Function Spaces
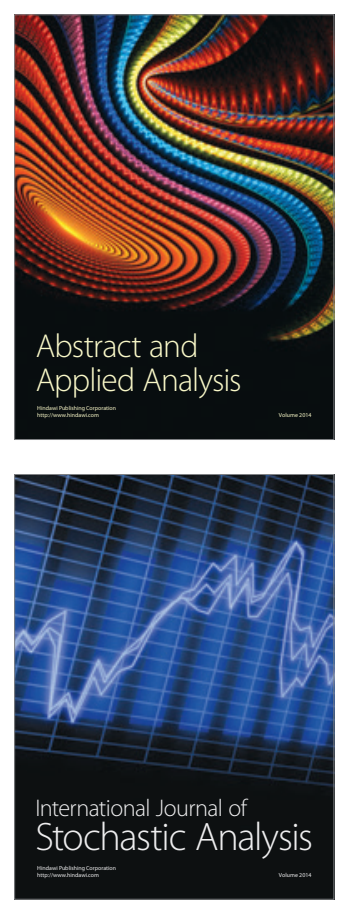

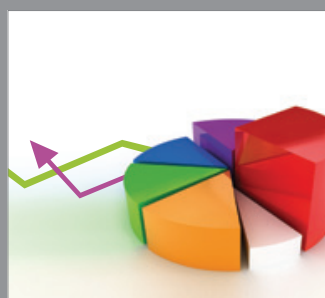

ournal of

Probability and Statistics

Promensencen
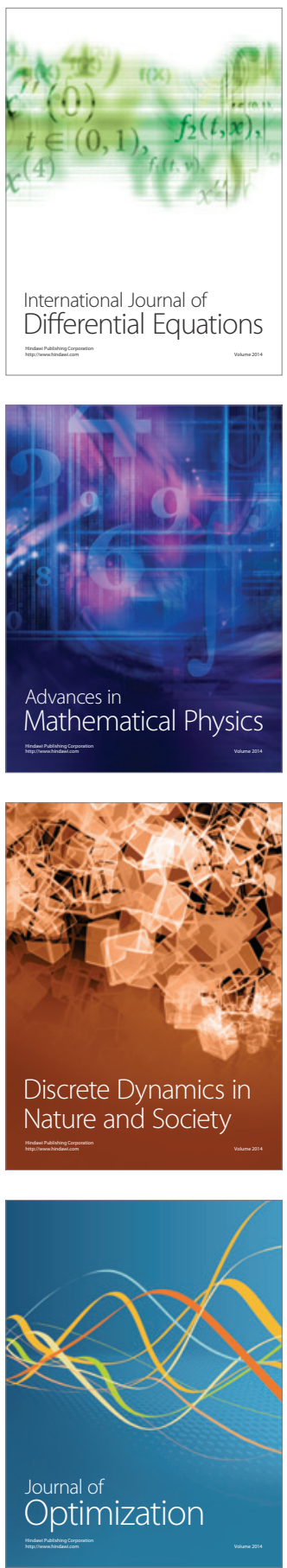